\title{
Pontine Infarct Presenting with Atypical Dental Pain: A Case Report
}

\author{
Rajat Goel ${ }^{1}$, Sanjeev Kumar ${ }^{2}$, Ajay Panwar ${ }^{3, *}$ and Abhishek B. Singh ${ }^{4}$ \\ ${ }^{1}$ Department of Endodontics, SGT Dental College Hospital and Research Institute, Gurgaon, India; ${ }^{2}$ Department of \\ Medicine, Lala Lajpat Rai Memorial Medical College, Meerut, India; ${ }^{3}$ Department of Neurology, Vardhman Mahavir \\ Medical College and Safdarjung Hospital, New Delhi, India; ${ }^{4}$ Department of ENT, Uttar Pradesh Regional Institute of \\ Medical Sciences and Research, Saifai, India
}

\begin{abstract}
Orofacial pain' most commonly occurs due to dental causes like caries, gingivitis or periodontitis. Other common causes of 'orofacial pain' are sinusitis, temporomandibular joint(TMJ) dysfunction, otitis externa, tension headache and migraine. In some patients, the etiology of 'orofacial pain' remains undetected despite optimal evaluation. A few patients in the practice of clinical dentistry presents with dental pain without any identifiable dental etiology. Such patients are classified under the category of 'atypical odontalgia'. 'Atypical odontalgia' is reported to be prevalent in $2.1 \%$ of the individuals. 'Atypical orofacial pain' and 'atypical odontalgia' can result from the neurological diseases like multiple sclerosis, trigeminal neuralgia and herpes infection. Trigeminal neuralgia has been frequently documented as a cause of 'atypical orofacial pain' and 'atypical odontalgia'. There are a few isolated case reports of acute pontine stroke resulting in 'atypical orofacial pain' and 'atypical odontalgia'. However, pontine stroke as a cause of atypical odontalgia is limited to only a few cases, hence prevalence is not established. This case is one, where a patient presented with acute onset atypical dental pain with no identifiable dental etiology, further diagnosed as an acute pontine infarct on neuroimaging. A 40 years old male presented with acute onset, diffuse teeth pain on right side. Dental examination was normal. Magnetic resonance imaging(MRI) of the brain had an acute infarct in right pons near the trigeminal root entry zone(REZ). Pontine infarct presenting with dental pain as a manifestation of trigeminal neuropathy, has rarely been reported previously. This stresses on the importance of neuroradiology in evaluation of atypical cases of dental pain.
\end{abstract}

Keywords: Pontine stroke, Trigeminal neuropathy, trigeminal neuralgia.

\section{INTRODUCTION}

Orofacial pain is very common with an estimated prevalence of about $10 \%$ in adults and $50 \%$ in elderly population [1]. Commonly, acute orofacial pain is of odontogenic origin, dental caries being the most frequent cause [2]. However, acute orofacial pain also, frequently results due to a number of non-odontogenic causes namely sinusitis, cervical spondylosis, otitis externa, sialadenitis and temporomandibular joint (TMJ) dysfunction [3]. There can be several causes of chronic orofacial pain like local inflammation (gingivitis, pulpitis, periodontitis), nociceptive (cancer, osteoarthritis), vascular (Migraine), muscular (tension headache), neurological (trigeminal neuralgia, multiple sclerosis, postherpetic) and idiopathic (atypical odontalgia, idiopathic facial pain). Trigeminal neuralgia has been frequently classified as 'Idiopathic' when no apparent cause of trigeminal root entry zone involvement is found like demyelination, tumor or vascular compression. 'Atypical odontalgia' presents with acute onset severe pain in teeth with no recognizable odontogenic cause. There is a famous case history of a case of 'atypical odontalgia' being diagnosed as 'Trigeminal neuralgia' who underwent trigeminal ganglion decompression surgery without any improvement [4]. This case

*Address correspondence to this author at the Department of Neurology, Vardhman Mahavir Medical College and Safdarjung Hospital, New Delhi, India; Tel: +917499171227; E-mail: ajay19panwar@gmail.com highlights the degree of confusion which a pain of trigeminal nerve origin can create in the practice of clinical dentistry. While trigeminal neuralgia is characterized by episodic lancinating, triggerable pains with intermittent pain free intervals, trigeminal neuropathic pain, on the other hand, is constant, non-triggerable and unremitting. Patients with trigeminal neuralgia are known to have minimal compression and demyelination at the root entry zone(REZ) of the trigeminal nerve while trigeminal neuropathy results from a definite injury to the trigeminal nerve. Trigeminal neuropathy is a well recognized disorder which may manifest as sensory loss in facial regions innervated by trigeminal nerve, or may cause weakness of jaw muscles. It is a rare entity and exact incidence and prevalence is not known. The symptoms usually occur as a result of injury of the trigeminal nerve and possibly, by the transection of central trigeminal pathways. The multiple etiologies for trigeminal neuropathy can be trauma, tumors, infectious or demyelinating diseases, diseases of the connective tissue or even idiopathic origin [5]. Trigeminal neuropathy, at times, can be the first manifestation of a tumor, hence important for timely diagnosis [6]. The onset of symptoms can be acute(trauma), subacute(infection) or chronic( tumors, connective tissue disorders), depending on the etiology [7]. However, sudden onset of symptoms are extremely rare and usually seen in vascular events. This case presents trigeminal neuropathy with a sudden onset dental pain, the cause being acute pontine stroke, a rarity in terms of etiology, mode of onset and symptoms. 
There are anecdotal case reports of acute pontine stroke presenting as trigeminal neuropathy or atypical orofacial pain, exact incidence and prevalence however, is not known.

\section{CASE REPORT}

A 40 years old male patient presented at our dental out patient department with acute onset dental pain for past 6 hours. It was restricted to right upper and lower teeth in a diffuse pattern. There was no history of dental caries, trauma or prior dental treatment. The patient was a known hypertensive for past 4 years on irregular medications. On examination, pulse was $84 / \mathrm{min}$, regular. Blood pressure was $140 / 90$ $\mathrm{mm} \mathrm{Hg}$. Dental examination revealed no abnormality. Orthopantomogram(OPG) [Sirona Orthophos XG] revealed complete permanent dentition with no abnormality in the morphological and anatomical structures of maxilla, mandible and associated structures. (Fig. 1) Thus, the dental etiology for the pain being ruled out, he was referred for a neurological consultation. Neurological examination revealed decreased pain, temperature and touch sensations in maxillary and mandibular divisions of trigeminal nerve on right side. Muscles of mastication were however, normal. Rest of the neurological examination revealed no neurological deficit. On further investigations, routine hematological and biochemical investigations including blood sugar were normal. Magnetic resonance imaging (MRI) [GE Signa Excite 1.5T] of brain revealed an acute infarct in right lateral pons close to root entry zone (REZ) of trigeminal nerve. (Fig. 2) Magnetic resonance angiography(MRA) of intracranial with neck vessels and two dimensional echocardiography(2-D Echo) was normal. Patient's therapy was started with asprin $150 \mathrm{mg}$ once a day and atorvastatin $20 \mathrm{mg}$ once a day. He was also put on perindopril and indapamide combination, with advice of regular blood pressure monitoring. Pain responded substantially to carbamazepine $200 \mathrm{mg}$ twice a day. Patient was followed up after 2 weeks with significant symptomatic improvement.

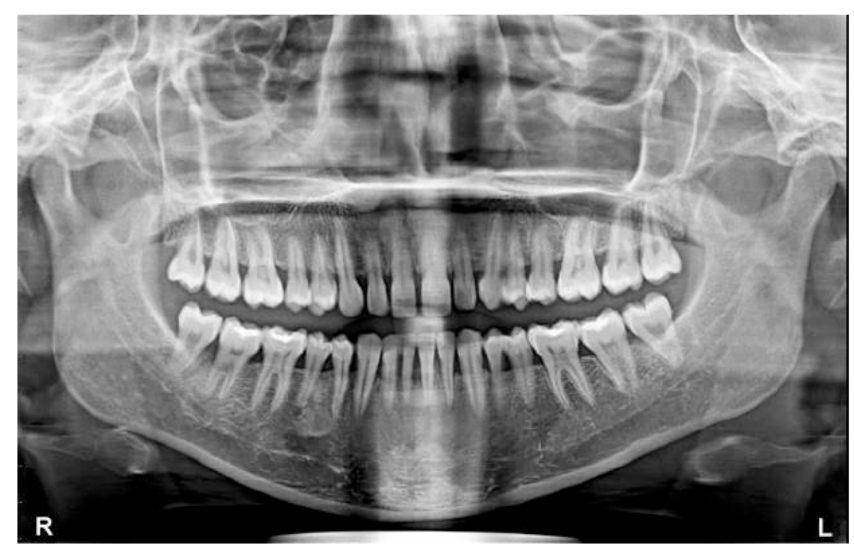

Fig. (1). OPG (Sirona Orthophos XG) reveals complete permanent dentition with no abnormality in the morphological and anatomical structures of maxilla, mandible and associated structures.

\section{DISCUSSION}

This is a classical case of acute onset 'Atypical odontalgia' where a patient with a neurological etiology presents to the faculty of clinical dentistry. The diagnostic confusion, was settled by neuroimaging(Brain MRI), showing an acute pontine infarct near the trigeminal root entry zone(REZ), thus establishing trigeminal neuropathy secondary to pontine infarct as the cause of 'atypical odontalgia'. Trigeminal neuralgia and trigeminal neuropathy secondary to demyelination, vascular aberrations and tumors at the trigeminal REZ are frequently reported causes of 'atypical orofacial pain' and 'atypical odontalgia'. Pontine infarct is a rare cause of trigeminal neuropathy. Kinoshita et al. reported two cases of isolated sensory trigeminal neuropathy secondary to pontine infarct at REZ of trigeminal nerve [8]. Katsuna also reported pontine infarct with a similar presentation [9]. Peker reported trigeminal neuralgia secondary to pontine infarct transecting central trigeminal pathways [10]. Some authors have reported trigeminal neuralgia secondary to ipsilateral pontine infarct in the vicinity of trigeminal nerve REZ [11]. Trigeminal neuropathy commonly presents with loss of sensation on face or weakness of jaw muscles. Trigeminal neuropathic pain, is persistent with no intermittent pain free intervals, and without any trigger factors in contrast to trigeminal neuralgia, where it is episodic, triggerable and remitting. Pain in both the conditions is restricted to face [12]. Trigeminal neuropathy manifesting with atypical dental pain, as in our patient, has been reported rarely. Barring a few case reports, exact prevalence is not known. So, this case presents an uncommon etiology of secondary trigeminal neuropathy with an uncommon presentation.

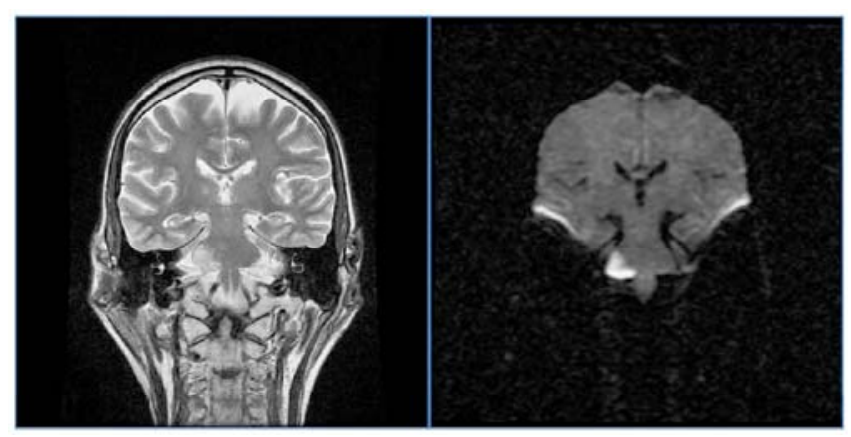

(A)

(B)

Fig. (2). T2-weighted coronal MR imaging (A) shows hyperintense signal in right pons near the root entry zone(REZ) of trigeminal nerve which shows corresponding diffusion restriction on DWI coronal image (B), confirming of acute pontine infarct.

Transection of central trigeminal pathways is implicated in pontine infarction causing trigeminal neuropathy [10]. The precise mechanism is however, not defined. King hypothesized that it is the hyperreactivity of trigeminal nucleus in response to electrical activity of peripheral trigeminal nerve, that results in the symptoms of trigeminal neuropathy in these cases [13]. List and Williams said that it is a pathologic multineuronal reflex of the trigeminal system in the brainstem that causes the symptoms [14]. However, some authors have made a consensus statement that it is the demyelination of the trigeminal pathways, secondary to the lesion, which results in ephaptic transmission and spontaneous firing of the trigeminal nerve, that causes the symptoms $[9,10]$. However, these electrophysiological changes in the trigeminal system need some time to develop(usually in a few days), while in our case, patient developed symptoms of 
trigeminal neuropathy at the onset.So,these mechanisms are unlikely to explain the physiological basis of symptoms in this case. An alternative mechanism that has been proposed is infarction of trigeminal sensory nucleus and spinal trigeminal nucleus, which can be the probable explanation in our case.

This case where, a primarily neurological disease presented as a dental outpatient and ultimately diagnosed with the aid of MRI brain, highlights the importance of neuroradiology in the clinical practice of Oro-maxillofacial dentistry.

\section{CONCLUSION}

Dental pain is an uncommon presentation of trigeminal neuropathy. One must be aware of it so as to avoid the misdiagnosis in those rare cases, where dental examination and investigations turn out to be normal. Also, this case highlights the importance of Neuroradiology in a few atypical cases in clinical dentistry.

\section{CONFLICT OF INTEREST}

The authors confirm that this article content has no conflict of interest.

\section{ACKNOWLEDGEMENTS}

Declared none.

\section{REFERENCES}

[1] Madland G, Feinmann C. Chronic facial pain: a multidisciplinary problem. J Neurol Neurosur Psychiatry 2001; 71(6): 716-9.

[2] Scully C, Shotts R. Mouth ulcers and other causes of orofacial soreness and pain. BMJ 2000; 321(7254): 162-5.

[3] Siccoli MM, Bassetti CL, Sándor PS. Facial pain: clinical differential diagnosis. Lancet Neurol 2006; 5(3): 257-67.

[4] Lilly JP, Law AS. Atypical odontalgia misdiagnosed as odontogenic pain: A case report and discussion of treatment. J Endodont 1997; 23(5): 337-9.

[5] Flint S, Scully C. Isolated trigeminal sensory neuropathy: a heterogeneous group of disorders. Oral Sur Oral Med Oral Pathol 1990; 69(2): 153-6.

[6] Penarrocha M, Cervello MA, Marti E, Bagan JV. Trigeminal neuropathy. Oral Dis 2007; 13(2): 141-50.

[7] Goto H, Matsuo H, Fukudome T, Shibuya N, Ohnishi A, Nakamura H. Chronic autonomic neuropathy in a patient with primary Sjogren's syndrome. J Neurol Neurosurg Psychiatry 2000; 69(1): 135.

[8] Kinoshita Y, Harada A, Yasukouchi H, Tsuru E, Okudera T. Isolated trigeminal sensory neuropathy due to pontine infarction of the root entry zone--report of two cases. Brain Nerve 2008; 60(2): 1759.

[9] Katsuno M, Teramoto A. Secondary trigeminal neuropathy and neuralgia resulting from pontine infarction. J Stroke Cerebrovasc Dis. 2010; 19(3): 251-2.

[10] Peker S, Akansel G, Sun I, Pamir NM. Trigeminal neuralgia due to pontine infarction. Headache 2004; 44(10): 1043-5.

[11] Kim JB, Yu S. Trigeminal neuralgia after pontine infarction affecting the ipsilateral trigeminal nerve. J Neurol Neurosurg Psychiatry 2013; 84(8): 881-2.

[12] Burchiel KJ. Trigeminal neuropathic pain. Acta Neurochir Suppl 1993; 58: 145-9.

[13] King RB. Evidence for a central etiology of tic douloureux. 1967. J Neurosurg 2007; 107(1): 232-7.

[14] List CF, Williams JR. Pathogenesis of trigeminal neuralgia; a review. AMA Arch Neurol Psychiatry 1957; 77(1): 36-43.

(C) Goel et al.; Licensee Bentham Open.

This is an open access article licensed under the terms of the Creative Commons Attribution Non-Commercial License (http://creativecommons.org/licenses/by-nc/3.0/) which permits unrestricted, non-commercial use, distribution and reproduction in any medium, provided the work is properly cited. 